\title{
Optimization of Linear Switched Reluctance Motor for Single Neuron Adaptive Position Tracking Control based on Fruit Fly Optimization Algorithm
}

\author{
Xu Ke ${ }^{1}$, Tang Chuansheng ${ }^{2}$, Yang Jie ${ }^{1,3^{*}}$, Zhang Jiyang ${ }^{1}$ and Hu Junzhe ${ }^{4}$ \\ ${ }^{1}$ Henan Institute of Technology, Xinxiang Henan 453000, China \\ ${ }^{2}$ Nanyang Institute of Technology, Nanyang Henan 473004, China \\ ${ }^{3}$ Henan Key Equipment Engineering Research Center for New Energy Power Generation, Xinxiang Henan 453000, China \\ ${ }^{4}$ Kaiserslautern Technical University, Kaiserslautern Rhineland-Pfalz 67663, Germany
}

Received 14 October 2019; Accepted 31 December 2019

\begin{abstract}
A linear switched reluctance motor (LSRM) drive system is a complex and strongly coupled nonlinear system with time-varying parameters, friction and external disturbances. An intelligent position tracking control based on fruit fly optimization algorithm (FOA) was proposed in this study to effectively solve the effects of parameter uncertainty, load disturbance, thrust fluctuation, and friction on the performance of the LSRM system. A mathematical model of LSRM was constructed on the basis of its structure and characteristics, and spatial discretization was conducted. A single neuron adaptive controller was designed for the discretized LSRM, and its parameters were adjusted and optimized online using the FOA. The accuracy of the model was verified through experiments. Results show that the propose controller has high tracking (rotor position steady-state error is less than $10 \%$ ) and strong anti-interference performance (restores to the desired rotor position instruction in $0.01 \mathrm{~s}$ ). The proposed controller can better track the rotor position when the rotor position instruction changes, with smaller overshoot (less than 10\%) and can control the PID up to 30\% compared with proportional-integral-derivative (PID) controllers. The proposed method has high position tracking accuracy and strong robustness to external disturbances.
\end{abstract}

Keywords: LSRM, single neuron, FOA, adaptive

\section{Introduction}

A linear switched reluctance motor (LSRM) is a device that directly converts electrical energy in a straight-line direction. Traditional linear transportation needs to be accomplished using rotating motor and conversion device, which has high complexity, large amounts of noise, serious energy loss, slow speed, and poor precision. The LSRM is lightweight, has simple structure, and good control, and directly depends on the electromagnetic force between the primary and secondary to achieve linear motion. The overall cost of LSRM is higher compared with the linear driving system of rotating motor and rack. LSRMs have been increasingly applied with the rapid development of electronic switch devices and digital control technology. The LSRM is obtained through the radial expansion of the rotating switched reluctance motor, and its structure is a double convex pole. The stator stage has neither winding nor permanent magnet, and the stator guide rail is stacked by strip-shaped silicon steel with $0.5 \mathrm{~mm}$ thickness. The actuator has concentrated winding with aluminum profile, small inertia, and good magnetic circuit isolation effect. Three identical windings are installed on the movers and are staggered at $120^{\circ}$ in space [1]. The LSRM is a good alternative to direct linear drive system. It has been successfully applied in rail transit trains, unmanned aerial

*E-mail address: yangti56@163.com

ISSN: 1791-2377@ 2020 School of Science, IHU. All rights reserved.

doi:10.25103/jestr.131.21 vehicle electromagnetic ejection device, deep mine hoist system, cordless direct drive elevators in urban highrise/super-high-rise buildings and 3D high-rise/deep urban garages. The LSRM meets the requirements of high speed and high precision in modern industry because of its simple structure, high reliability, low starting current, large starting thrust, and large acceleration, making it suitable for the need of straight line feed movement (no worm gear worm, rolling screw, gear rack and other intermediate transmission links), which has been widely investigated by scholars all over the world [2-3].

Thus, the LSRM is a complex strong coupling nonlinear system with double convex machine structure, which has a strong coupling relationship between phase current, flux linkage, and rotating speed, and makes the flux, inductance, and torque, which are nonlinear functions, vary with the position of the mover, thereby making it highly nonlinear [45]. The actuator winding of LSRM is embedded in the slot, and the grooving effect causes thrust fluctuations on the actuator [6]. Some factors, such as parameter uncertainty, load disturbance, thrust fluctuation, and friction, increase the design difficulty of the LSRM control system [7].

\section{State of the art}

Realizing high-performance dynamic control of motors under multifactor constraints has been widely investigated to overcome complex factors, such as strong coupling, nonlinear, and multitime variation [8-20]. Proportional- 
Xu Ke, Tang Chuansheng, Yang Jie, Zhang Jiyang and Hu Junzhe/

\section{Journal of Engineering Science and Technology Review 13 (1) (2020) 160 - 165}

integral-derivative (PID) controllers occupy a dominant position in the existing industrial control field because its structure is simple and easy to implement. Maslan et al. [8] applied a PID controller to an LSRM by combining three different heuristic optimization techniques namely, PIDparticle swarm optimization (PSO), fuzzy PSO, and genetic algorithm (GA)-PSO. GA and PSO were applied to the control system for reducing large torque fluctuations, accelerating rising time, and avoiding interference affecting the driving performance. Their proposed control can meet the requirements of high accuracy and dynamic performance. However, the PID controller strongly depends on the system model, thereby it cannot achieve highperformance control. Ghoulemallah et al. [9] also combined the above mentioned heuristic optimization techniques and applied GA and particle colony optimization on a control system to reduce large torque fluctuation, accelerate rise time, and avoid interference affecting driving performance. However, the number of fuzzy rules and the size of particle cluster optimization cannot be determined. Li et al. [10] applied fuzzy control theory to the design of the PID controller and presented the fuzzy PID and fuzzy PID control of the variable domain to improve the suppression ability of system parameters and external disturbances. The appropriate number of fuzzy rules needs to be determined. The control performance is poor when the rules are excessively few, whereas the performance requires a long time period when the rules are excessive. Modern control methods, such as adaptive, sliding mode, backstepping, and passive control, have been applied to the position tracking control of motors [11-19]. Ammar et al. [11] adopted an adaptive control strategy, namely, model reference adaptive controller (MRAC) to adjust the torque and stator flux of asynchronous motors to ensure robust control and small sensitivity of machine parameters compared with traditional PI controllers. Moreover, an adaptive speed and load torque observer was designed on the basis of Lyapunov stability and the control performance was improved by reducing the number of sensors, thereby reducing the cost of the global control system and improving its reliability. The proposed controller was verified through MATLAB/Simulink simulation test and real-time interface experiment using a dSpace 1104 board. This method can effectively realize sensorless control and parameter adaptive online estimation. However, it still depends on the initial state of the motor and the parameters of the reference model, and the adaptive control needs to estimate the control parameters online, thereby consuming a large amount of time. Pal et al. [12] proposed an MRAC through golden segmentation. They reported that the instantaneous and steady-state values of virtual resistance (r) in the reference and adaptive models are not affected by the change in stator resistance. The MRAC has the unique form of instantaneous reactive and steadystate reactive power to completely eliminate any flux estimation requirements during velocity estimation. Therefore, this method is insensitive to drift and saturation of the integrator. Thus, it can be used to accurately estimate the zero speed or near the zero speed. The performance of the proposed R-MRAC driver under different speed ranges and modes was verified on MATLAB/Simulink. The proposed method depends on the accuracy of system model parameters and increases the calculation time. Tang et al. [13] estimated the stator current online with sliding mode control using a linear motor model in $\alpha \beta$ static coordinate system and the position and velocity of the mover using a back EMF model. Their method can effectively avoid the influence of external disturbances on the speed and position estimation accuracies of the stator. However, the dependence on stator resistance is strong. A load disturbance observer was designed by Ali et al. [14] to improve the inherent "buffeting" phenomenon caused by traditional sliding mode control to motor drive systems, thereby effectively improving the performance of system control. This method has high estimation accuracy when the motor is running at high speed but cannot meet the requirements at low-speed operation. Karami-Mollaee et al. [15] proposed a new observer design method and dynamic sliding mode control to solve the uncertain position control problem of servo induction motors. Although the "buffeting" phenomenon can be effectively improved by placing the integrator (or lowpass filter) into the system control input, the augmented system using dynamic sliding mode control is still 1D larger than the actual system (when the integrator is used, and the object model should be completely known. Thus, Ali et al. proposed a new adaptive state observer to maintain the controllability and positioning accuracy of the system under external load torque change. The error of the observer converges to zero, and the system satisfies Lyapunov stability theory through derivation. The simulation results verified the effectiveness of the proposed method. Although this method could improve the buffeting phenomenon of the system and improve its dynamic performance to a certain extent, it increases the complexity of the controller design and reduces the system reliability to a certain extent. Harrouz et al. [16] used PI control, backstepping method, and adaptive backstepping control to control the speed of DC motors. Simulation was conducted on MATLAB Simulink software, and the overall performance of adaptive backstepping control was compared with conventional PI and backstepping control. The proposed method combines adaptive control with backstepping control to realize the reverse design and stability analysis of the controller. However, this method needs to derive the system model many times, thereby resulting in "computational explosion" caused by derivation. Therefore, the above methods were combined with other methods. Tang et al. [17] extended adaptive backstepping control to the thrust and magnetic chain ring of linear motor and realized the integrated design of speed loop, thrust, and magnetic chain ring. However, the design of position loop causes "computational explosion" during derivation. Zhao et al. [18] applied passive control to an LSRM, which is equivalent to a passive port system. Passive control theory was used to design the speed and position control of the system. This method heavily depends on the resistance, inductance, and other parameters of the motor. Chebaani et al. [19] proposed and implemented a sensorless finite state predictive torque control system based on direct torque control (DTC) to improve its performance. The system uses DTC to extend Kalman filter as driver for induction motor model state estimation and system sensorless control. The simulation and experimental results indicated that the driving system using this technology could effectively reduce the flux linkage and torque pulsation with good dynamic and steady-state performance. This method needs prior data and model parameters to predict the state variables of the system, and the size of predictive control sample data has a great influence on the accuracy of the system. Siamak et al. [20] combined fuzzy control with sliding mode control to realize automatic adjustment of control gain through fuzzy estimation and compared it with PID and fuzzy controllers to verify its superiority. The method has the self-learning ability of fuzzy control and the 
Xu Ke, Tang Chuansheng, Yang Jie, Zhang Jiyang and Hu Junzhe/

Journal of Engineering Science and Technology Review 13 (1) (2020) 160 - 165

insensitivity of sliding mode control to the time-varying parameters, thereby improving the buffeting of the system. However, the buffeting phenomenon, which affects the system performance, cannot be fully eliminated. Qiu et al. [21] proposed a controller by converting the control problem of LSRM in the presence of network delay, Qiu et al. [21] converted into a leading matrix inequality. However, the proposed controller ignores the influence of time-varying parameters and disturbances. Wang et al. [22] proposed a second-order trajectory smoothing filter tracking control strategy by combining feedforward and linear feedback control. This method effectively improves the buffeting of the system and simplifies the controller solution. However, the controller structure remains complex.

In recent years, variable structure control has been increasingly used because of its simple structure, easy implementation, and insensitivity to system parameter changes and external disturbances. Single neuron control is a simple intelligent control strategy that can effectively solve the problem of designing the structure of complex neural networks, and has been widely investigated [23-24]. Xiao et al. [23] used single neurons to current control of $d$ and $q$ axes of permanent magnet synchronous motor for realizing dynamic current tracking. However, the neural network weights of this method are difficult to determine. Butt et al. [24] used single neurons for the speed tracking control of IPMSM and verified them through simulation. Their proposed method has good dynamic performance with the changes in parameters and external disturbances. Realizing the online adjustment of neural network weights requires to be investigated to improve system performance.

This study presented a single neuron adaptive control based on fruit fly optimization algorithm (FOA) for the position tracking of LSRM. Considering the weight adjustment rules existing in traditional single neuron control are easy to fall into the local best ones, the FOA [25-26] was used to adjust the neuron weight online for improving the self-learning and adaptive abilities of neuron and realizing the high-performance position tracking control of LSRM.

The remainder of this study is organized as follows. Section 3 describes the structure of LSRM, constructs the calculation model of voltage, motion, and thrust of LSRM, and designs the single neuron adaptive controller based on the FOA. Section 4 analyzes the thrust, mover, and rotor characteristics of the model using the proposed single neuron adaptive control method based on the FOA, and obtains the expected thrust control output curve, response comparison curve of the mover position, and rotor position response. Section 5 summarizes the study and provides relevant conclusions.

\section{Methodology}

\subsection{Structure and mathematical model of the LSRM}

The structure of the studied LSRM studied is shown in Fig. 1. The motor is a three-phase single-side switch reluctance motor, which is similar to a rotary electric machine in a radial direction. The rotor and stator of the rotating motor correspond to the actuator and stator of the LSRM, respectively. The parameters of the structural model are defined in Table 1.

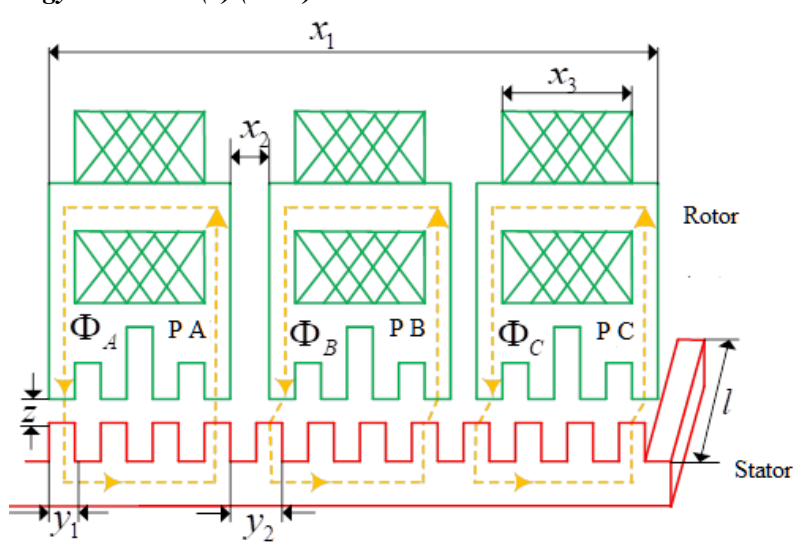

Fig. 1. Structural model of the LSRM

Table 1. Model parameters of the LSRM

\begin{tabular}{|c|c|c|c|c|c|}
\hline Parameter & Meaning & Unit & Parameter & Meaning & Unit \\
\hline$x_{1}$ & $\begin{array}{l}\text { Length of } \\
\text { rotor }\end{array}$ & $m m$ & $y_{1}$ & $\begin{array}{c}\text { Width of } \\
\text { pole }\end{array}$ & $\mathrm{mm}$ \\
\hline$x_{2}$ & $\begin{array}{l}\text { Spacing of } \\
\text { winding }\end{array}$ & $m m$ & $y_{2}$ & $\begin{array}{l}\text { Spacing } \\
\text { of pole }\end{array}$ & $\mathrm{mm}$ \\
\hline$x_{3}$ & $\begin{array}{l}\text { Width of } \\
\text { winding }\end{array}$ & $m m$ & $l$ & $\begin{array}{l}\text { Length of } \\
\text { pole }\end{array}$ & $\mathrm{mm}$ \\
\hline$z$ & $\begin{array}{l}\text { Distance } \\
\text { of air gap }\end{array}$ & $\mathrm{mm}$ & & & \\
\hline
\end{tabular}

The mathematical model of the LSRM without the influence of mutual inductance between windings can be expressed as follows:

Voltage is expressed as:

$U_{k}=R_{k}+d \lambda_{k} / d t, k=a, b, c$

Thrust expression is expressed as:

$F=\sum_{k=1}^{3} \frac{\partial \lambda_{k}(x, i)}{\partial x} i_{k}$

Motion is expressed as:

$F=M_{n} \ddot{x}+B_{n} \dot{x}+F_{L}+F_{f}+F_{r}$

Where $U_{k}, i_{k}, \lambda_{k}$ and $R_{k}$ are the phase voltage, phase current, phase flux and phase resistance, respectively; $M_{n}$ is the quality of rotor; $x$ is the displacement of rotor; $\dot{x}=v$ is the velocity of rotor; $F$ is the electromagnetic thrust; $B_{n}$ is the linear friction coefficients; $F_{L}, F_{f}$ and $F_{r}$ are the thrust fluctuation, load disturbance and nonlinear friction of the system, respectively.

\subsection{Discretization of the LSRM}

The motion equation of the LSRM needs to be discretized to design the outer loop controller of position and velocity.

The transfer function of the system is obtained using Formula (3):

$G(s)=X(s) / F(s)=1 /\left(M_{n} s^{2}+B_{n} s\right)$

As shown in Formula (4) the system is a second-order when the sampling time is $T_{s}=1 \mathrm{~ms}$, and the $z$ transform is used to discretize the system. The discrete object after $z$ transform is expressed as: 
Xu Ke, Tang Chuansheng, Yang Jie, Zhang Jiyang and Hu Junzhe/

Journal of Engineering Science and Technology Review 13 (1) (2020) 160 - 165

$$
\begin{aligned}
y(k)= & -a_{1} y(k-1)-a_{2} y(k-2)+b_{1} u(k-1) \\
& +b_{2} u(k-2)+d(k)
\end{aligned}
$$

Where $a_{1}, a_{2}, b_{1}$ and $b_{2}$ are the discrete coefficients, and $d(k)$ is the total disturbances of the system.

\subsection{Design of single neuron adaptive controller}

The proposed controller structure is shown in Fig. 2. The single neuron adaptive controller is composed of single

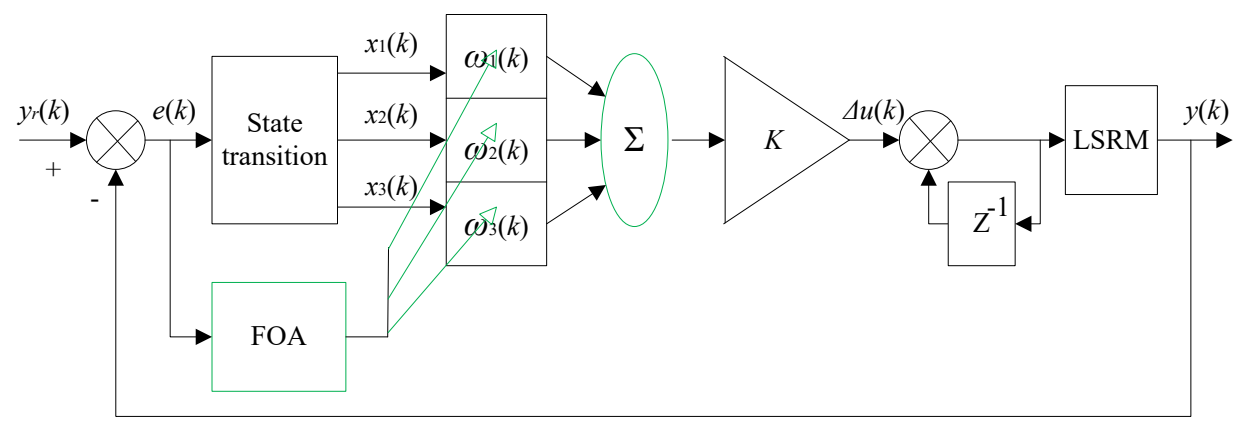

Fig.2. Single neuron adaptive controller for LSRM

neuron adaptive control and online optimization of neuron weights. The self-adaptive and self-organizing functions are realized through the automatic adjustment of neuron weight coefficients (online fruit fly optimization). The control rate is expressed as follows:

$$
u(k)=u(k-1)+K \sum_{i=1}^{3} \omega_{i}(k) x_{i}(k)
$$

Where $u(k)$ and $u(k-1)$ are the outputs of the controller (expected electromagnetic thrust) in $k$ and $k-1$, and $\omega_{i}(\mathrm{i}, 2,3)$ are the weight coefficients of $x_{i}(\mathrm{i}, 2,3)$, which are expressed as follows:

$$
\left\{\begin{array}{l}
x_{1}(k)=e(k) \\
x_{2}(k)=e(k)-e(k-1) \\
x_{2}(k)=\Delta^{2} e(k)=e(k)-2 e(k-1)+e(k-2)
\end{array}\right.
$$

Where $e(k), e(k-1)$ and $e(k-2)$ are the position tracking errors at times of $k, k-1$ and $k-2$, respectively.

The weight coefficient of single neuron is determined using the FOA, the optimized performance index is taken as $J=\frac{1}{2}\left(y_{r}(k)-y(k)\right)^{2}$, and the specific optimization is shown in Fig.3.

In this algorithm, the random direction and distance of the fruit fly can be determined using Formula (8):

$$
\left\{\begin{array}{l}
X_{i}=X_{0}+h * \operatorname{rand}() \\
Y_{i}=Y_{0}+h * \operatorname{rand}()
\end{array}\right.
$$

Where $X_{0}$ and $Y_{0}$ are the initial distances on the corresponding coordinates, $h$ is the step size, and $X_{i}$ and $Y_{i}$ are the distances from origin $i$, Thus, the function of distance $D_{i}$ and taste concentration function $S_{i}$ are calculated as follows:

$$
\left\{\begin{array}{l}
D_{i}=\operatorname{sqrt}\left(X_{i}^{2}+Y_{i}^{2}\right) \\
S_{i}=1 / D_{i}
\end{array}\right.
$$

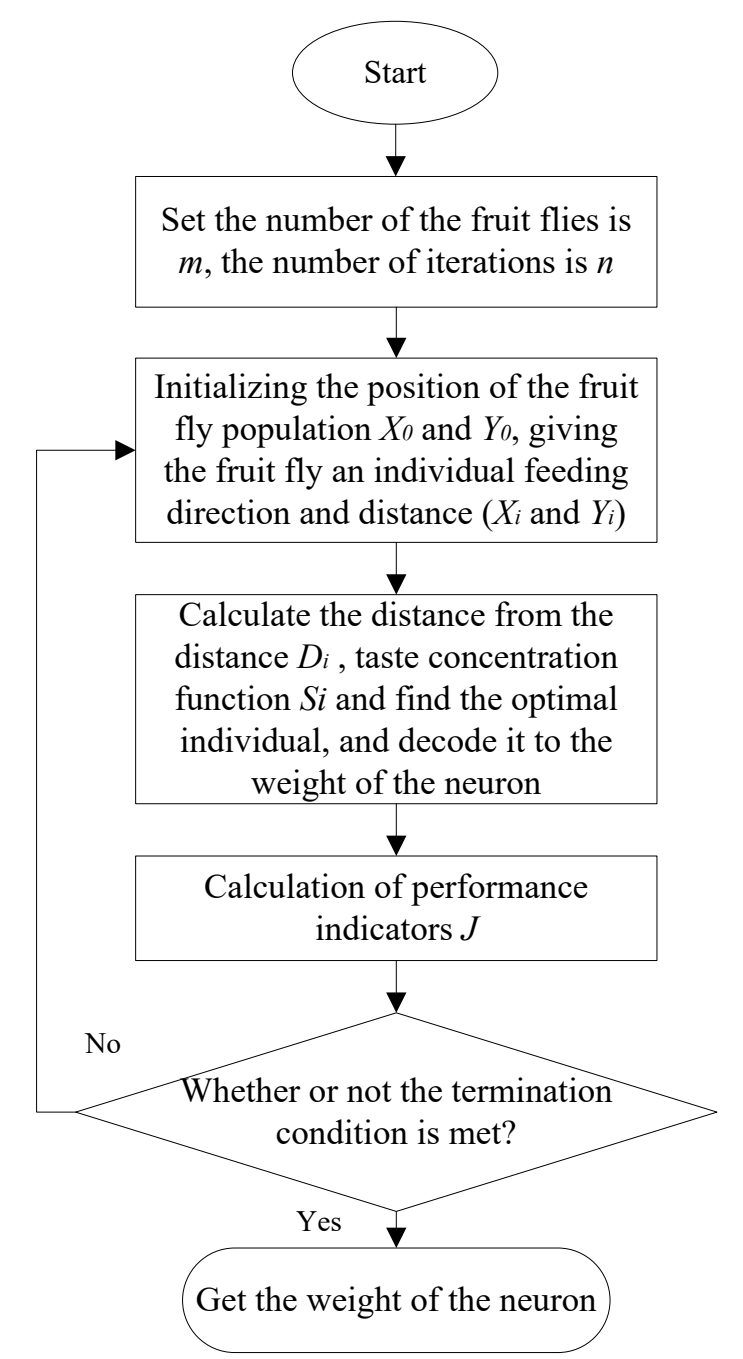

Fig.3. Parameter optimization of drosophila algorithm based on single neuron adaptive control

\section{Result Analysis and Discussion}

The proposed method is compared with a PID control to verify its effectiveness. 
Xu Ke, Tang Chuansheng, Yang Jie, Zhang Jiyang and Hu Junzhe/

Journal of Engineering Science and Technology Review 13 (1) (2020) 160 - 165

The main parameters of the LSRM are expressed as follows: the quality of rotor is $M_{n}=4.6 \mathrm{~kg}$, the coefficient of friction is $B_{n}=0.01 \mathrm{~N} \cdot \mathrm{m} / \mathrm{s}$, the sampling period of system discretization is $T_{s}=1 \mathrm{~ms}$, the total simulation time is $1 \mathrm{~s}$, the command signal is a trapezoidal wave with the amplitude of 1 , and a sudden load is added in $t=0.2 \mathrm{~s}$ and $t=0.7 \mathrm{~s}$. The parameter setting of the controller is expressed as follows: the neuron proportion coefficient is set as $K=0.1$, the initial weight value of the neuron is $W=\left[\omega_{1}(0) \omega_{2}(0) \omega_{3}(0)\right]=[0.10 .10 .1]$, the number of iterations of the FOA is 100 , the population size is 100 , and the random direction and distance interval are $[-1,1]$. The simulation results are shown in Fig. 4-Fig. 7, and Fig. 8 shows the comparison of the tracking performance between the PID control and the proposed controller.

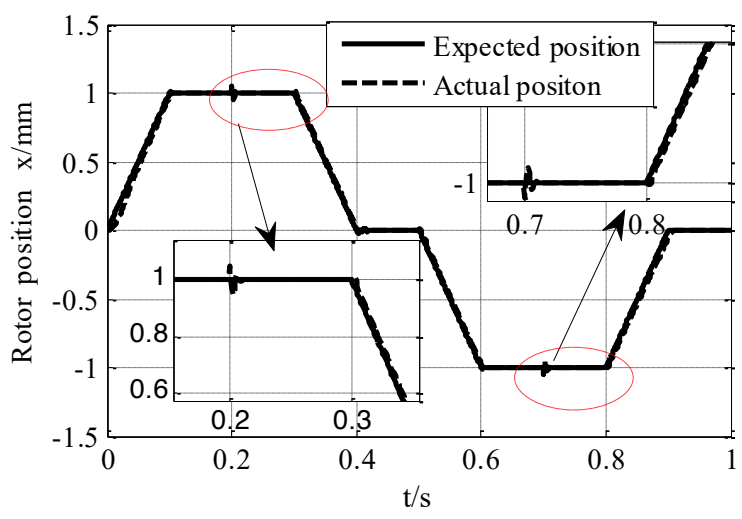

Fig.4. Rotor position response curve of the LSRM

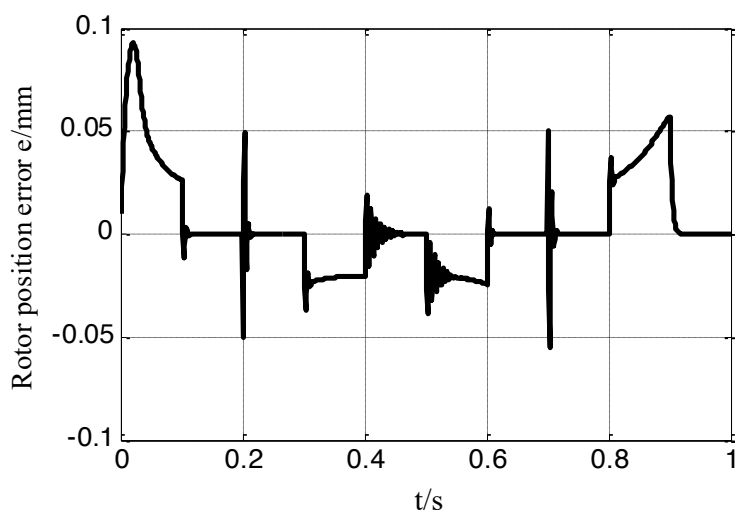

Fig.5. Rotor position error curve of the LSRM

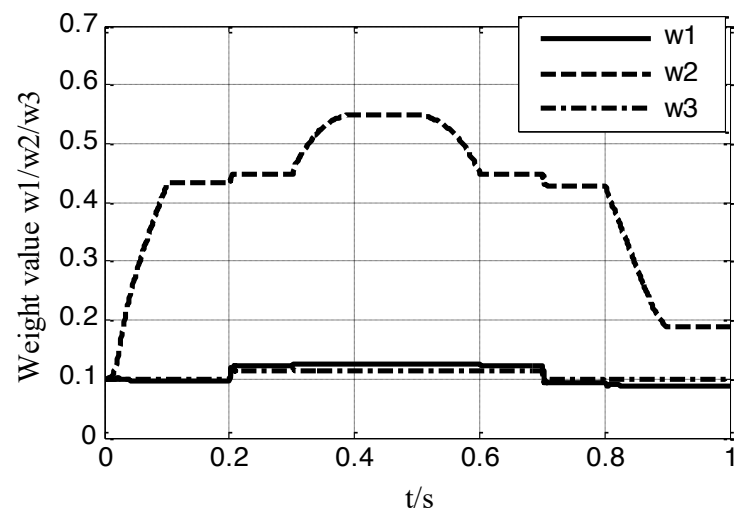

Fig.6. Optimization curve of fruit fly with single neuron weight

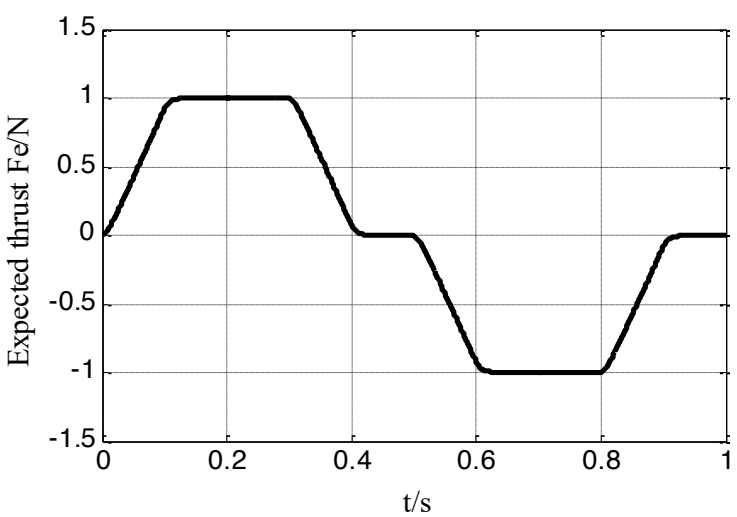

Fig.7. Curve of expected thrust control output

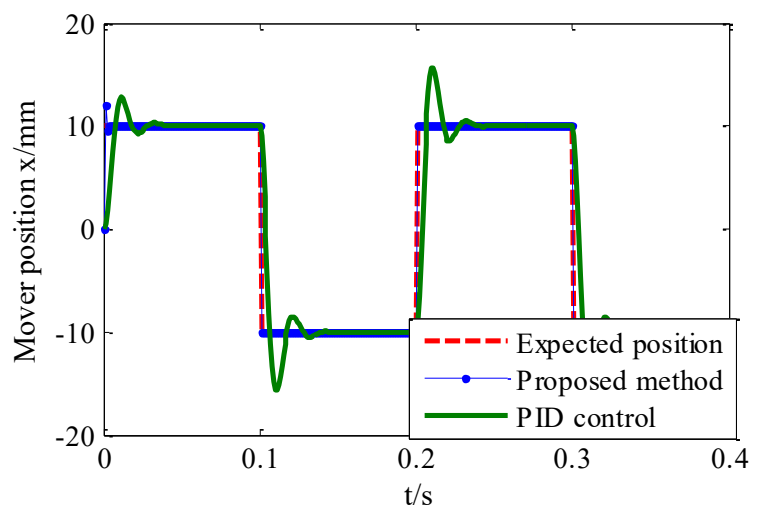

Fig.8. Comparison response curve of rotor position

As shown in Fig. 4-Fig. 8, the following observations are summarized as: (1) In the start-up stage, the proposed method has good dynamic tracking ability, where the dynamic tracking error is controlled within $10 \%$, and the tracking accuracy is extremely high in steady state. (2) At 0.2 and $0.7 \mathrm{~s}$, the rotor position error increases to $5 \%$ and recovers to the specified position within $0.01 \mathrm{~s}$ with the addition of external disturbance. (3) As shown in Fig. 6, the weights of neurons can be automatically adjusted on the basis of the change in rotor position, and the dynamic response ability of the system can be improved using the FOA. (4) The output of the controller (expected thrust) is a smooth curve that can effectively improve the torque performance. Compared with the conventional PID control, the proposed single neuron adaptive control has better dynamic startup performance. The PID control has serious overshoot when the rotor position immensely changes.

The proposed strategy can quickly and stably track the system position instructions and has strong robustness to external disturbances.

\section{Conclusions}

A new position control strategy of LSRM was proposed in this study to explore the influences of uncertain factors, such as time-varying parameters and external disturbances, on the accuracy of LSRM. The mathematical model of LSRM was combined with single neuron control theory and FOA. The conclusions through theoretical derivation and numerical simulation are summarized as follows:

(1) The proposed control system has high steady-state tracking performance, and its steady-state tracking error is less than $2 \%$ (as shown in Fig. 5). 
Xu Ke, Tang Chuansheng, Yang Jie, Zhang Jiyang and Hu Junzhe/

\section{Journal of Engineering Science and Technology Review 13 (1) (2020) 160 - 165}

(2) The proposed control system has strong antiinterference and can quickly return to the specified position under large load disturbances and external interferences.

(3) The output of the controller, that is, expected electromagnetic thrust, has small thrust fluctuation, and exhibits a smooth curve.

(4) The proposed control system has strong adaptive ability, and its control parameters are adjusted online through the optimization of FOA.

This study is mainly based on the physical model of LSRM. A rotor position tracking scheme that can effectively overcome the changes in system parameters and external disturbances is proposed by combining theory and numerical simulation, which can meet the high-speed and highprecision requirements of servo systems, and as certain application value. The proposed control scheme needs to be verified and optimized through subsequent experiments because of the limited experimental conditions, especially the influence of mutual inductance on the system (secondary factors) is ignored in the physical LSRM model. Future studies should investigate the influence of this factor on the experimental platform.

\section{Acknowledgements}

This work was supported by the Key Scientific Research Projects of Higher Education Institution of Henan Province (Grant No. 20B470003 and Grant No. 18B470007) and Key R\&D and the Promotion Special Project of Scientific Research Program of Henan Province Grant No. $202102210084)$.

This is an Open Access article distributed under the terms of the Creative Commons Attribution License

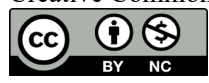

\section{References}

1. Li, K., "Switching phrase linear motor and its control". Guangzhou: South China University of Technology, China, 2009, pp. 2-12.

2. Li, S. Y., Cheng, K. W. E., Zhu, J. W., Zou, Y., "Design and application of a decoupled rotary-linear switched reluctance motor for concentrated photovoltaic power generation". Institution of Engineering and Technology Electric Power Applications, 12(7), 2018, pp. 908-915.

3. Zhang, G. L., Liu, G. H., Gao, C. X., "The design and analysis of the superconducting linear switched reluctance motor for maglev train”. Cryogenics \& superconductivity, 46(5), 2018, pp. 51-55.

4. Guo, F., Geng, B. M., Zhang, R. F., "Mathematical modeling of linear switched reluctance motor with transverse magnetic field". Electric Motor and Control, 18 (10), 2014, pp. 42-49.

5. Chen, H., Xu, S., Wei, W. K., Yang, J., Nie, R., "Reliability assessment of double-sided linear switched reluctance generator system based on hierarchical markov model". IEEE Transactions on Industrial Electronics, 66(6), 2019, pp. 4901-4911.

6. Sun, H. T., Chen, Y., Chang, X. M., "Design and analysis of permanent magnetic switching reluctance linear motor". Journal of Taiyuan University of Technology, 48(2), 2017, pp. 232-236.

7. Zhou, L. F., Zhao, S. W., "Position control of linear switched reluctance motor based on fast nonsingular terminal sliding mode". Small \& Special Electrical Machines, 45(8), 2017, pp. 52 -56.

8. Maslan, M. N., Kokumai, H., Sato, K., "Development and precise positioning control of a thin and compact linear switched reluctance motor". Precision Engineering, 48, 2016, pp. 256-278.

9. Ghoulemallah, B., Sebti, B., Abdesselem, C., Said, B., "Genetic algorithm and particle swarm optimization tuned fuzzy PID controller on direct torque control of dual star induction motor". Journal of Central South University, 26(7), 2019, pp. 1886-1896.

10. Li, D., Zhang, T., Tang, C. S., "Study on variable domain fuzzy PID control technology for linear permanent Magnet synchronous Motor". Machine tool and hydraulic pressure, 40(15), 2012, pp. $27-$ 29.

11. Ammar, A., Benakcha, A., Bourek, A., "Adaptive MRAC-based direct torque control with SVM for sensorless induction motor using adaptive observer". The International Journal of Advanced Manufacturing Technology, 91(5-8), 2017, pp. 1631-1641.

12. Pal, A., Kumar, R., Das, S., "Sensorless Speed Control of Induction Motor Driven Electric Vehicle Using Model Reference Adaptive Controller". Energy Procedia, 90, 2016, pp. 540-551.

13. Tang, C. S., Dai, Y. H., Xiao, Y., "High precision position control of PMSLM using adaptive sliding-mode approach". Journal of Electrical Systems, 10(4), 2014, pp. 456-464.

14. Ali, N., Rehman, A. U., Alam, W., Maqsood, H., "Disturbance Observer Based Robust Sliding Mode Control of Permanent Magnet Synchronous Motor". Journal of Electrical Engineering \& Technology, 14(6), 2019, pp. 2531-2538.
15. Karami-Mollaee, A., Tirandaz, H., Barambones, O., "Dynamic sliding mode position control of induction motors based load torque compensation using adaptive state observer". Compel, 37(6), 2018, pp. 2249-2262.

16. Harrouz, A., Becheri, H., Colak, I., Kayisli, K., "Backstepping control of a separately excited DC motor". Electrical Engineering, 100(3), 2018, pp. 1393-1403.

17. Tang, C. S., Duan, Z. Y., "Direct thrust-controlled PMSLM servo system based on back-stepping control". Institute of Electrical Engineers of Japan Transactions on Electrical and Electronic Engineering, 13(5), 2018, pp. 785-790.

18. Zhao, S. W., Cheung N. C., Gan, W. C., Yang, J. M., Zhang, Q., "Passivity-based control of linear switched reluctance motors with robustness consideration". Electric Power Applications, 2(3), 2008, pp. 164-171.

19. Chebaani, M., Goléa, A., Benchouia, M. T., Goléa, N., "Sensorless finite-state predictive torque control of induction motor fed by fourswitch inverter using extended Kalman filter". Compel, 37(6), 2018, pp. 2006-2024.

20. Siamak, M., Soltanpour, M. R., Abdollahi, H., "Adaptive fuzzy control method for a linear switched reluctance moto". Institution of Engineering and Technology Electric Power Applications, 12(9), 2018, pp. 1328-1336.

21. Qiu, L., Shi, Y., Zhang, B., Pan, J., Lai, X., “Tracking Control of Networked Multiple Linear Switched Reluctance Machines Control System Based on Position Compensation Approach". IEEE Transactions on Industrial Informatics, 14(12), 2018, pp. 53685377.

22. Wang, J., Zeng, Q. M., Zhang, Z., "Second-order discrete smoothing filter tracking control of linear switched reluctance motor". Proceedings of the Chinese Society for Electrical Engineering, 11, 2004, pp. 179-184.

23. Xiao, Y. T., Chen, B. H., "Study on the decoupling control of the output double-closed-loop neurons of the frequency converter". Acta Engergiae Solaris Sinica, 37(6), 2016, pp. 1367-1372.

24. Butt, C. B., Rahman, M. A., "Intelligent Speed Control of Interior Permanent Magnet Motor Drives Using a Single Untrained Artificial Neuron". IEEE Transactions on Industry Applications, 49(4), 2013, pp. 1836-1843.

25. Pi, J., Ma, S., Zhang, Q. Q., Wang, L. P., Cui, D. Z., "Prediction model of exhaust temperature of GRNN aero-engine based on improved fruit fly algorithm". Journal of Aerospace Power, 34(1), 2019, pp. 8-17.

26. Yang, X. Y., Chen, Q. Y., Wang, M., Zhang, L., "Optimal control of wind power and hydropower cooperative Drosophila melanogaster considering network constraints". Proceedings of the Chinese Society for Electrical Engineering, 37(18), 2017, pp. 5286-5293, 5527. 